Manuelle Medizin 2020 · 58:182 https://doi.org/10.1007/s00337-02000682-6

(c) Springer Medizin Verlag $\mathrm{GmbH}$, ein Teil von Springer Nature 2020

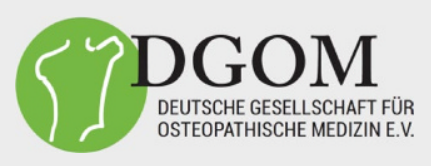
DEUTSCHE GESELLSCHAFT FÜR
OSTEOPATHISCHE MEDIZIN E.V.

\section{Deutsche Gesellschaft} für Osteopathische Medizin (DGOM) e.V.

\author{
Goethestraße 18 \\ 68161 Mannheim \\ Tel.: 0621/43630268 \\ Fax: 0621/4363 0269 \\ Internet: kontakt@dgom.info \\ www.dgom.info
}

V.i.S.d.P.

Dr. med. Ute Beckmann

Berliner Str. 40

63654 Büdingen

\section{Kursprogramm 2020 (Auszug)}

\section{Sämtliche Kurse finden Sie unter www.dgom.info}

Aufgrund der Corona-Pandemie musste auch die DGOM einige Fortbildungskurse absagen. In der aktuellen Situation bei Drucklegung können wir leider nicht garantieren, dass die Kurse wie angegeben stattfinden; im Gegenzug kommen noch Nachholtermine hinzu. Aktuelle Informationen unter www.dgom.info.

\section{Auszug aus dem Grundcurriculum}

\section{GKOM/T}

20.06. -21.06 .2020$

05.09.-06.09.2020

20.11.-22.11.2020

Berlin

Freiburg

Boppard (OV 1)

\section{MET 1}

18.09.-20.09.2020

Mannheim

\section{MET 2}

09.10.-11.10.2020

Mannheim

\section{MET 3}

02.10.-04.10.2020

Mannheim

\section{MFR 1}

28.08.-30.08.2020

Mannheim

\section{MFR 2}

26.06.-28.06.2020

29.11.-01.12.2020

Boppard (OV 6)

Mannheim

Anatomie (Schwangere können nicht teilnehmen)

12.09.-13.09.2020

Düsseldorf

\section{Cranio 1}

11.09.-13.09.2020

Mannheim

VIS 1

25.09.-27.09.2020

Mannheim

Cost

21.08.-23.08.2020
Mannheim
Func 1

$\begin{array}{ll}\text { 19.06. }-21.06 .2020 & \text { Mannheim } \\ \text { 09.10.-11.10.2020 } & \text { Berlin }\end{array}$

\section{Parietale Integration}

30.10.-01.11.2020 Boppard (OV 8)

\section{Einsteigerkurse für Mediziner aller Fachrichtungen}

Möchten Sie sich unverbindlich über die osteopathische Medizin informieren? Hier lernen Sie symptomorientiert verschiedene Therapieansätze kennen und beleuchten funktionelle Verkettungen anhand des faszialen, muskuloskelettalen, viszeralen und kranialen Systems.

Ziel ist es, eine osteopathische Herangehensweise mit Ihrem ärztlichen Wissen zu verknüpfen und entsprechende Therapieansätze und Lösungen in Ihren Alltag einzubinden. Sie können die Intensivkurse unabhängig voneinander buchen. Bei Beginn einer Ausbildung bei der DGOM rechnen wir die Teilnahme an mind. zwei Kursen als Grundkurs an.

Schwindel, Cephalgien, Cervikobrachialgien

03.10.-04.10.2020 Mannheim

Sie möchten osteopathische Techniken erlernen, aber Ihnen fehlen noch die manuellen Grundlagen? Im integrierten Curriculum von DGOM und DGMSM erlernen Sie beides in einem abgestimmten, flexiblen Zeitrahmen. Nähere Informationen und das komplette Kursprogramm unter www. dgom.info oder rufen Sie uns an: 0621-4363 0268

Kursprogramm Osteopathische Verfahren in Kooperation mit der Ärztekammer Westfalen-Lippe

Die DGOM und die Ärztekammer Westfalen-Lippe bieten als Kooperationspartner die strukturierte curriculäre Fortbildung "osteopathische Verfahren" (OV) an.

Die Fortbildung im Rahmen von 160 Stunden baut auf der vorhandenen Zusatzweiterbildung „Manuelle Medizin“ auf. Zweck ist es, fertige Manualmediziner ins Thema einzuführen und einige dieser Verfahren vertieft zu behandeln.

Weitere Informationen finden Sie auf unserer Homepage www.dgom. info. 